\title{
Unfavourable TB Outcomes in PLHIV with Delayed TB Treatment Initiation-A Retrospective Analysis
}

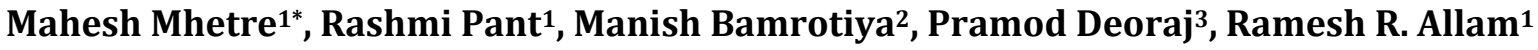 \\ ${ }^{1}$ SHARE INDIA, Ghanpur Village, Medchal, India \\ ${ }^{2}$ National AIDS Control Organization (NACO), New Delhi, India \\ ${ }^{3}$ Maharashtra State AIDS Control Society, Mumbai, India \\ Email: ^maheshmhetre@shareindia.org, rashmi.pant@shareindia.org, bamrotiya.manish@gmail.com,dnrtwi@gmail.com, \\ rame05allam@gmail.com
}

How to cite this paper: Mhetre, M., Pant, R., Bamrotiya, M., Deoraj, P. and Allam, R.R. (2021) Unfavourable TB Outcomes in PLHIV with Delayed TB Treatment Initiation-A Retrospective Analysis. Open Journal of Epidemiology, 11, 293-302. https://doi.org/10.4236/ojepi.2021.113026

Received: May 21, 2021

Accepted: August 21, 2021

Published: August 24, 2021

Copyright $\odot 2021$ by author(s) and Scientific Research Publishing Inc. This work is licensed under the Creative Commons Attribution International License (CC BY 4.0).

http://creativecommons.org/licenses/by/4.0/ (c) (i) Open Access

\begin{abstract}
Background \& Objectives: Timely initiation of anti-tubercular treatment (ATT) is key to obtain favourable outcomes among people living with HIV (PLHIV) co-infected with tuberculosis (TB). There is little evidence to predict treatment outcome and the delay in initiation of ATT among co-infected. The analysis explores the relationship between different ATT initiation delay times and TB outcomes. Methods: Retrospective analysis of secondary data of HIV-TB co-infected patients attending two antiretroviral therapy (ART) clinics in Pune district of Maharashtra, India. Logistic regression models were used to calculate risk. Results: Of the total 457 co-infected PLHIV initiated on ATT from December 2016 to June 2018, there were 298 who had completed TB treatment. Outcomes of ATT were available for 243 patients. Of the 243 patient's records analysed, $61 \%$ were males and $\leq 11 \%$ were less than 18 years. $20 \%$ were previously treated. The risk of unfavourable outcomes increased monotonically from $\mathrm{OR}=1.1$, to $\mathrm{OR}=1.73$ for $>2$ days to $>7$ days delay in ATT initiation respectively. Males $(\mathrm{OR}=2.11)$, newly initiated patients $(\mathrm{OR}=1.54)$ are exposed to higher risk of unfavourable TB outcomes when ATT initiation is delayed beyond seven days. Conclusion: Risk of unfavourable outcomes increase in delay time for ATT initiation. Patient characters should be considered for prioritised referral and availability of test results on the same day of diagnosis to foster same day ATT initiation. More analysis on larger data sets with richer clinical information will generate confirmatory evidence.
\end{abstract}

\section{Keywords}

HIV-TB Co-Infection, ATT Initiation, TB Outcome 


\section{Introduction}

India has an estimated 2.14 million people living with HIV (PLHIV) and 15\% of total PLHIV is contributed by Maharashtra state [1]. Maharashtra has HIV prevalence of $0.33 \%$ [1]. Incidence of $\mathrm{TB}$ in India is 2.7 million while the mortality due to TB is 0.4 million [2]. TB numbers in India. In 2018, 192,458 TB cases were notified in Maharashtra and 3778 were notified from Pune of which $8 \%$ were constituted by PLHIV [2].

Government of India introduced fixed dose daily Anti-TB Treatment (ATT) at the ART centres for PLHIV from the hitherto alternate day regimen delivered through Directly observed therapy, short course (DOTS) centres as part of package of single window services for HIV-TB. The primary focus of National AIDS Control Program (NACP) is prevention and management of TB in PLHIV. National NACP and Revised National Tuberculosis Control Programme (RNTCP) jointly drafted the national guidelines for prevention and management of TB at Antiretroviral therapy (ART) Centres [3].

Studies from other countries that had integrated TB and HIV care, indicated an increase in favourable outcomes after Anti tubercular treatment (ATT) such as reduced mortality, treatment completion and sputum smear conversion rates [4] [5]. Studies indicated that early initiation of ART among HIV and TB co-infected PLHIV significantly decreased incidence of HIV disease progression. As per regional response plan of World Health Organisation (WHO) for TB-HIV-2017-2021 and also as per Revised National Tuberculosis Program (RNTCP), TB treatment initiation within 7 days of diagnosis is one of the core indicators to measure quality of TB care [1] [6]. There is paucity of evidence on the effect of delayed ATT initiation beyond 7 days on ATT outcomes in PLHIV.

This study aims to explore the association between delayed initiation of ATT on TB treatment outcomes among PLHIV. It also seeks to determine patient characteristics that predispose individuals who received delayed ATT, to unfavourable TB outcome, than those initiated early.

\section{Methods}

\subsection{Study Design and Setting}

We conducted an analytical cross-sectional exploratory study based on secondary data analysis. Secondary data was obtained from ART centres of Yashwantrao Chavan Memorial (YCM) Hospital and Sub District Hospital, Baramati of Pune district, Maharashtra, India. YCM ART centre is located in Pimpri Chinchwad Municipal Corporation while Sub-district Hospital, Baramati is located in rural Pune district. Both ART centres provide single window delivery services for HIV-TB co-infected cases. In March 2019, there are 6136 and 2296 PLHIV registered in active care at YCM and Sub District Hospital, Baramati ART centre respectively.

Guidelines on "Prevention and Management of TB in PLHIV at ART Centres" (2016) recommends verbal screening of all PLHIV for active TB and evaluated 
for TB using CBNAAT or appropriate diagnostic tools. One spot sputum sample is collection at Designated Microscopy Centre (DMC) for CBNAAT [3]. CBNAAT results and other clinical and diagnostic test results for TB diagnosis are available in digital or paper form format at ART centre and the Staff Nurse at ARTC makes a phone call to all PLHIV diagnosed of TB. Prior to initiation of ATT, the ART staff counsels patients on need for early initiation of treatment for favourable TB treatment outcomes and emphasize the need for adherence.

\subsection{Data Collection}

We extracted records of co-infected cases that were initiated on ATT from December 2016 to May 2018. Information on demographic details and clinical characteristics such as date of diagnosis, date of ATT and ART treatment initiation along with other details such as whether initiated on CPT or not was collected from HIV-TB register provided. The HIV-TB line list and HIV-TB register were verified to ensure completeness and correctness. $10 \%$ of the data was reviewed for data accuracy from these reference sources during Technical assistance (TA) site visits to the ART centres.

\subsection{Measurements}

The date of TB diagnosis is the date when TB disease was confirmed among the presumptive TB cases based on CBNAAT/clinical or other laboratory tests. The date of TB treatment initiation is the day when the confirmed case of TB was initiated on daily ATT.

Delay in ATT initiation was calculated as the difference between date of initiation and date of diagnosis. These differences were then dichotomized into separate variable at 1 to 7 days of delay.

Outcome of TB treatment after ATT completion (six months for Cat I regimen (new) while eight months for Cat II regimen) was available in HIV-TB register. Outcomes were initially categorized as favorable (cure, treatment completed) and unfavorable (died, transfer out, treatment failure and lost to follow-up). Age of patient dichotomized at eighteen years to distinguish between young and adult patient.

\subsection{Statistical Analysis}

Categorical variables were summarised as frequencies and percentages and are represented as column graphs. The distribution of TB treatment outcomes by gender, patient type and age group are represented using column graphs showing percentage. Exploratory logistic regression models were used to calculate the risk of unfavourable outcomes as odds ratios. The dependent variable in these models was binary TB outcome $(0=$ favourable and $1=$ unfavourable TB outcome) and the independent variable was delay in TB treatment initiation $(>1$ to $>7$ days). Delay in ATT initiation was calculated as the difference between date of diagnosis of TB and date of ATT initiation. This continuous variable was 
then recreated into several categorical variables describing different categories of delay. For example, the first categorisation was, $0=$ same day initiation and $1=$ Not same day, the second categorisation was, $0=$ same day initiation and $1=$ " $>1$ day delay", the third categorisation was, $0=$ same day initiation and $1=$ ">2 days delay" and so on The model was built in an iterative manner beginning with one day delay in ATT to $>7$ days delay. The results of these models are presented in Figure 2. To explore which characteristics were important to predispose patients at a higher risk of unfavourable outcomes due to delay in initiation beyond seven days, stratified analyses was done using logistic regression. Table 2 shows results from logistic regression models with TB outcome as dependent variable and delay in initiation (dichotomised at 7 days) as independent variable for each category of patient characteristics: age group $(\leq 18 />18)$, gender (male/female), patient type (previously treated/newly initiated). The risk measured were expressed as odd ratios (OR) and $95 \%$ confidence intervals. The reference category for delay in ATT initiation was $\leq 7$ days.

OR $>1$ is interpreted as higher odds of unfavourable TB outcome in $>7$ days ATT initiation delay as compared to $\leq 7$ days delay for that patient characteristic category.

To compare whether there was a significant difference in patient characteristics for those initiated on ATT on same day of diagnosis versus those not initiated on same day, chi-square test of independence was used. P-values were reported in Table 3 and are interpreted as significant differences in patient characteristics between the two groups if $\mathrm{p}$-value $<0.05$.

\subsection{Ethics and Approvals}

Approvals were obtained from National AIDS Control Organization (NACO), Maharashtra State AIDS control society (MSACS) and the Medicity Ethical Committee exempted the study from ethics review as this is a secondary data analysis. Data sheets were stripped of patient identifier information such as name and address. Each data row was assigned unique identity numbers.

\section{Results}

A total of 457 patient records of PLHIV initiated on ATT were extracted from two hospitals-YCM, Pune (290 (63.5\%)) and SDH, Baramati (167 (36.5\%)). Eight records were excluded as either the date of diagnosis or date of ATT initiation was absent. Of the remaining 449 patients, 159 (35.4\%) were on treatment and $54(12 \%)$ were transferred out. Treatment outcomes were available for 236 (52.6\%) PLHIV initiated on ATT. Of these 236 patients, 76 (32.2\%) had unfavourable outcomes (died $=68(28.8 \%)$, Loss to follow-up $(\mathrm{LFU})=7(3 \%)$, failed treatment $=1(0.4 \%))$ and $160(67.8 \%)$ had favourable outcomes (cured $=94$ $(39.8 \%)$, completed treatment $=66(28 \%))$ (Figure 1$)$. The median delay in ATT initiation before start of ART was 29 days with IQR of 14 to 57 days. The median delay in ATT initiation after start of ART was 259 days with IQR of 30 to 1310 days. 

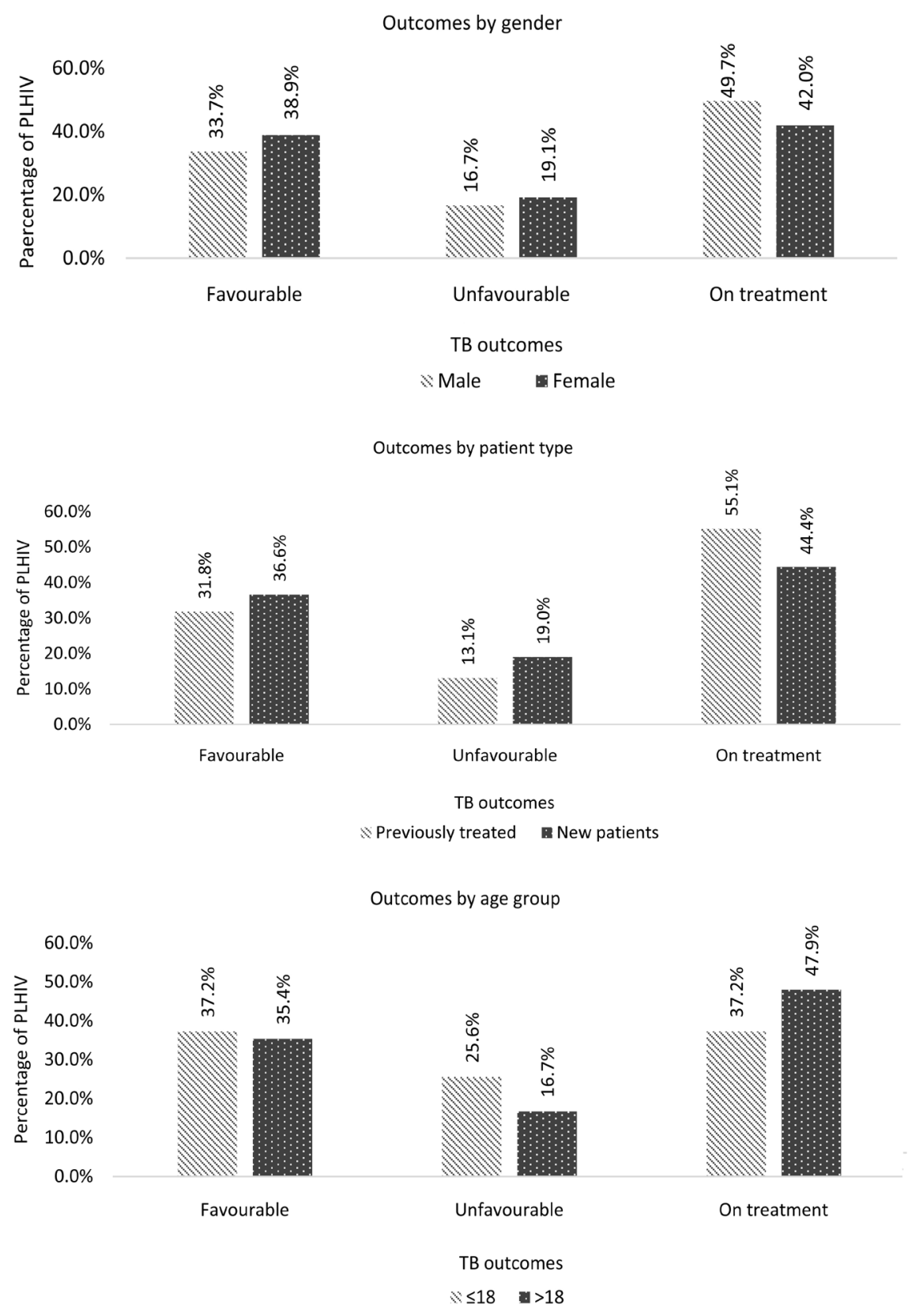

Figure 1. Distribution of $\mathrm{N}=457$ patients initiated on ATT by TB outcomes across age, gender and patient type categories.

Demographic and patient characteristics of patients (236) with TB outcomes are represented in Table 1. Majority 148 (61\%) were males, average age was 37.5 years with a standard deviation of 13.8 years and 27 (11\%) patients were below 19 years of age. Previously treated patients (recurrent, treated after loss to follow-up and treatment after failure) constituted $20 \%$. Only one center had records on type of TB and among these 55.2\% had pulmonary TB. Drug resistance was ruled out in 150 (66.2\%) and for 88 (32.6\%) it was unknown. Majority $(81.4 \%)$. were on category-I treatment and $76(32.2 \%)$ had unfavourable outcome (LFU 2.9\%, Died 26.8\% and Failure 0.4\%). 
Table 1. Characteristics of HIV-TB co-infected PLHIV.

\begin{tabular}{|c|c|c|}
\hline Characteristic & & $\mathrm{N}(\%)$ \\
\hline Mean age in years (mean \pm SD) & & $37.5 \pm 13.8$ \\
\hline \multirow{3}{*}{ Age group } & $\leq 18$ & $27(11.2 \%)$ \\
\hline & & \\
\hline & $>18$ & $215(88.8 \%)$ \\
\hline \multirow{2}{*}{ Sex } & Male & $148(61.2 \%)$ \\
\hline & Female & $94(38.8 \%)$ \\
\hline \multirow{2}{*}{ Type of patient } & Previously treated & $48(19.9 \%)$ \\
\hline & New & $193(80.1 \%)$ \\
\hline \multirow{2}{*}{ Type of TB } & Pulmonary TB & $73(55.3 \%)$ \\
\hline & Extra-pulmonary $\mathrm{TB}$ & $59(44.7 \%)$ \\
\hline \multirow{3}{*}{ Drug resistant status } & Yes & $3(1.2 \%)$ \\
\hline & No & $150(66.2 \%)$ \\
\hline & Unknown & $88(32.6 \%)$ \\
\hline \multirow{4}{*}{ ATT initiation } & Before ART & $139(36.3 \%)$ \\
\hline & Median days (IQR) & $29(14$ to 57$)$ \\
\hline & After ART & $244(63.7 \%)$ \\
\hline & Median days (IQR) & 259 (30 to 1310$)$ \\
\hline \multirow{2}{*}{ Outcomes } & Favourable & $160(67.8 \%)$ \\
\hline & Unfavourable & $76(32.2 \%)$ \\
\hline
\end{tabular}

Patients not initiated on ATT on day of TB diagnosis (same day) have 1.05 times higher odds of unfavourable outcomes than those initiated on same day. Although delay of more than 1 or 2 days shows decreased risk (OR $=0.92$ 0.94 ), the odds begin to increase monotonically from 1.1 to 1.73 if the delay in initiation exceeds three days. Among the $\leq 18$ years age group, the odds of unfavourable outcomes were 1.46 times higher for those delayed beyond 7 days than those initiated on ATT earlier. Similarly, males (OR $=2.11)$, newly initiated patients $(\mathrm{OR}=1.54)$ are exposed to higher risk of unfavourable TB outcomes when ATT initiation is delayed beyond seven days (Table 2). Figure 2 shows the risk of unfavourable TB outcomes for different delays in ATT initiation days. The graph shows an increasing risk of unfavourable outcomes as the delay in ATT initiation increases.

Comparison of profiles of patients initiated on ATT on same day versus those did not had no statistical significance. However very high percentage of those who experience some delay in ATT initiation, were new patients (83\%), males $(62.7 \%),>18$ years $(89.8 \%)$, category-I patients $(81.3 \%)$ and only $32.2 \%$ among these had unfavourable outcomes (Table 3 ). 


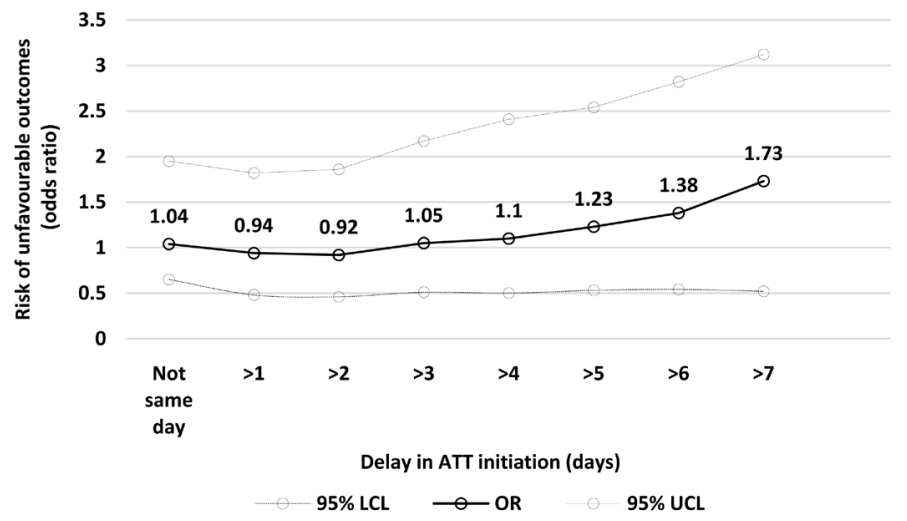

Figure 2. Risk of unfavourable TB outcomes among PLHIV corresponding to delay in number of days of ATT initiation after TB diagnosis.

Table 2. Odds ratio of unfavourable outcomes among patients with delayed ( $>7$ days) ATT initiation, stratified by specific patient characteristics.

\begin{tabular}{cccc}
\hline Characteristics & $\mathbf{N}$ & OR & 95\% CI \\
\hline Age group & & & \\
$\leq 18$ & 210 & 1.46 & $(0.6,3.6)$ \\
$>18$ & 24 & 0.43 & $(0.04,4.63)$ \\
Sex & & & \\
Male & 142 & 2.11 & $(0.76,5.9)$ \\
Female & 92 & 0.44 & $(0.09,2.2)$ \\
Patient type & & & \\
Previously treated & 43 & NA & NA \\
New & 190 & 1.54 & $(0.65,3.6)$ \\
\hline
\end{tabular}

${ }^{*}$ Cells had 0 frequencies. Hence odds ratios could not be calculated. ${ }^{* *}$ Reference category for independent variable, time to ATT initiation was $\leq 7$ days.

Table 3. Comparative profile of PLHIV initiated on ATT on same day versus those not initiated on same day.

\begin{tabular}{cccc}
\hline Characteristics & $\begin{array}{c}\text { Same day initiation } \\
\mathbf{1 7 5 ( 7 4 . 8 \% )}\end{array}$ & $\begin{array}{c}\text { Not initiated on same day } \\
\mathbf{5 9}(\mathbf{2 5 . 2 \% )}\end{array}$ & $\begin{array}{c}\text { Chi-sq. } \\
\text { p-value* }\end{array}$ \\
\hline Age group & & & \\
$\leq 18$ & $18(10.3 \%)$ & $6(10.2 \%)$ & 0.99 \\
$>18$ & $157(89.7 \%)$ & $53(89.8 \%)$ & \\
Gender & & & 0.83 \\
Male & $105(60 \%)$ & $22(37.3 \%)$ & \\
Female & $70(40 \%)$ & $10(17 \%)$ & 0.88 \\
Patient type & $33(19 \%)$ & $49(83 \%)$ & \\
Previously treated & $141(81 \%)$ & & $<0.0001$ \\
New & & $49(83 \%)$ & \\
DR-TB status & $98(56.3 \%)$ & $10(17 \%)$ & \\
Absent & $76(45.7 \%)$ & & 0.99 \\
Unknown & $120(68.6 \%)$ & $19(32.2 \%)$ & \\
Treatment outcomes & $55(31.4 \%)$ & & \\
Favourable & & & \\
Unfavourable & & & \\
\hline
\end{tabular}

${ }^{*} \mathrm{p}$-value from Fisher's exact test. 


\section{Discussion}

The exploratory analysis among HIV-TB co-infected PLHIV indicated a significant temporal relationship between incremental delay in ATT initiation and unfavourable ATT outcomes. This study has clearly demonstrated the recommendation of initiation of ATT within seven days of TB diagnosis. As per RNTCP guideline one of the core quality indictor is TB treatment initiation within 7 days for those found to be smear-positive [7]. In the study for TB treatment delay in Odisha, India, out of 1805 smear-positive patients there were $40 \%$ (721) who had a delay of more than 7 days. Significant delay was observed amongst HIV co-infected patients which were 6.2 times (95\%) [7].

Absence of $\mathrm{Tb}$ diagnosis services including previous history of ATT was attributed for TB treatment delay for $>7$ days in a study conducted in two districts of two states of India. In this study $>1 / 3$ with smear-positive had delayed TB treatment of $>7$ days after diagnosis [8]. A retrospective study among 330 MDR cases in Yangon Regional Tuberculosis Centre of Myanmar, in 2014 concluded that a delay in the initiation of MDR treatment was associated with poor treatment outcome. Out of 330 MDR cases, $81 \%$ achieved successful outcome. However, the study also highlighted that it is statistically insignificant considering other associated risk factors [5].

A cross sectional study among 735 new adult TB cases in Ethiopia indicated that $54 \%$ of the delay for TB treatment initiation was due to health system challenges. Out of these 735 cases, there were $68 \mathrm{HIV}$ positive cases. Among them there were $60 \%$ HIV positive individuals who had delay of $>25$ days while $40 \%$ delay of $<25$ days [9]. NACO 2018 technical guidelines for ART suggest that PLHIV with $<50$ CD4 cells should be initiated concomitant ART and ATT without delay [9]. A study among 64 pulmonary cases conducted in Patna, India for TB treatment delay of more than 7 days found that the treatment delay was similar among new TB cases (35\%) and retreatment cases (40\%) [10].

This study is the first to explore the association between delay in ATT initiation among PLHIV and TB treatment outcomes as per WHO guidelines in the NACP setting [11]. This exploratory study was done on small convenience sample. As a result the risk measured gave quantitative strength in association but did not yield statistical significance. A larger sample, more representative of the overall HIV-TB co-infected population will yield better insights. Additional information on clinical aspects such as baseline CD4, weight and adherence to ART and ATT will be beneficial in understanding the phenotypic pathways to TB treatment outcomes. This study specifically focuses on co-infected cases and signifies increase in unfavourable treatment outcome with delay for each day.

\section{Conclusion}

It is currently recommended in the Guidelines on "Prevention and Management of TB in PLHIV at ART Centres" (2016) for prompt TB treatment initiation irrespective of $\mathrm{CD} 4+\mathrm{T}$ cell count, but the optimal/ideal timing of ATT initiation 
after diagnosis needs to be specified. Considering that the sputum samples are tested on the same day after referral of presumptive TB cases and availability of Cartridge-based nucleic acid amplification test (CBNAAT) reports on the same day of testing, a need arises to ensure that they are initiated on TB treatment at the earliest or within a gap of seven days of TB diagnosis as recommended in the WHO regional response plan for TB-HIV (2017-2021) which clearly states anti-TB treatment initiation within seven days as one of the monitoring and evaluation indicators for HIV-TB to measure quality of TB care. The findings highlight the need for treatment initiation within 7 days for all diagnosed cases of TB through different diagnostic tests to get favourable outcomes and for preventing the spread of disease.

\section{Study Highlights}

- Our study confirmed that there is a major role of time in TB treatment initiation on getting favourable TB treatment outcome.

- History of TB treatment and gender was independently associated with an increased risk of unfavourable TB treatment outcome.

- Addition of clinical information such as CD count and adherence will strengthen the evidence for early initiation of ATT.

\section{Conflicts of Interest}

The authors declare no conflicts of interest regarding the publication of this paper.

\section{References}

[1] National AIDS Control Organisation (2017) India Estimations 2017 HIV, Fact Sheets.

[2] World Health Organization (2018) India Tuberculosis Report 2018. World Health Organization, Geneva.

[3] National AIDS Control Organisation (2016) Prevention of Tuberculosis among People Living with HIV at ART Centres.

[4] Dheda, K., Shean, K., Zumla, A., Badri, M., Streicher, E.M., Page-Shipp, L., Willcox, P., John, M.A., Reubenson, G., Govindasamy, D. and Wong, M. (2010) Early Treatment Outcomes and HIV Status of Patients with Extensively Drug-Resistant Tuberculosis in South Africa: A Retrospective Cohort Study. The Lancet, 375, 1798-1807. https://doi.org/10.1016/S0140-6736(10)60492-8

[5] Htun, Y.M., Khaing, T.M., Aung, N.M., Yin, Y., Myint, Z., Aung, S.T., Soonthornworasiri, N., Silachamroon, U., Kasetjaroen, Y. and Kaewkungwal, J. (2018) Delay in Treatment Initiation and Treatment Outcomes among Adult Patients with Multidrug-Resistant Tuberculosis at Yangon Regional Tuberculosis Centre, Myanmar: A Retrospective Study. PloS ONE, 13, e0209932.

[6] Regional Office for South-East Asia, World Health Organization (2017) Regional Response Plan for TB-HIV-2017-2021. WHO Regional Office for South-East Asia. https://apps.who.int/iris/handle/10665/254824

[7] Ilangovan, K., Nagaraja, S.B., Ananthakrishnan, R., Jacob, A.G., Tripathy, J.P. and 
Tamang, D. (2015) TB Treatment Delays in Odisha, India: Is It Expected Even after These Many Years of RNTCP Implementation?. PloS ONE, 10, e0125465. https://doi.org/10.1371/journal.pone.0125465

[8] Paul, D., Busireddy, A., Nagaraja, S.B., Satyanarayana, S., Dewan, P.K., Nair, S.A., Sarkar, S., Ahmed, Q.T., Sarkar, S., Shamrao, S.R. and Harries, A.D. (2012) Factors Associated with Delays in treatment Initiation after Tuberculosis Diagnosis in Two Districts of India. PloS ONE, 7, e39040.

https://doi.org/10.1371/journal.pone.0039040

[9] National AIDS Control Organisation (2018) National Technical Guidelines on Anti-Retroviral Treatment.

[10] Mistry, N., Lobo, E., Shah, S., Rangan, S. and Dholakia, Y. (2017) Pulmonary Tuberculosis in Patna, India: Durations, delays, and Health Care Seeking Behaviour among Patients Identified through Household Surveys. Journal of Epidemiology and Global Health, 7, 241-248. https://doi.org/10.1016/j.jegh.2017.08.001

[11] Sinha, S., Shekhar, R.C., Singh, G., Shah, N., Ahmad, H., Kumar, N., Sharma, S.K., Samantaray, J.C., Ranjan, S., Ekka, M. and Sreenivas, V. (2012) Early versus Delayed Initiation of Antiretroviral Therapy for Indian HIV-Infected Individuals with Tuberculosis on Antituberculosis Treatment. BMC Infectious Diseases, 12, 168.

https://doi.org/10.1186/1471-2334-12-168 